\title{
Lymphocytic Interstitial Pneumonitis: An Unusual Presentation of X-Linked Hyper Ig M Syndrome
}

\author{
Mohsen Reisi, Gholamreza Azizi, ${ }^{2,3}$ Tooba Momen, ${ }^{4}$ Hassan Abolhassani, ${ }^{3,5}$ and Asghar \\ Aghamohammadi, \\ ${ }^{1}$ Child Growth and Development Research Center, Pediatric Pulmonology Department, Research Institute of Primordial Prevention of Non- Communicable Disease, Isfahan \\ University of Medical Sciences, Isfahan, IR Iran \\ ${ }_{2}^{2}$ Imam Hassan Mojtaba Hospital, Alborz University of Medical Sciences, Karaj, IR Iran \\ ${ }_{3}^{3}$ Research Center for Immunodeficiencies, Pediatrics Center of Excellence, Children's Medical Center, Tehran University of Medical Sciences, Tehran, IR Iran \\ ${ }_{4}^{2}$ Pediatric immunology, Allergy and Asthma Department, Child Growth and Development Research Center, Research Institute of Primordial Prevention of Non-Communicable \\ Disease, Isfahan University of Medical Sciences, Isfahan, IR Iran \\ ${ }^{5}$ Division of Clinical Immunology, Department of Laboratory Medicine, Karolinska University, Stockholm, Sweden \\ ${ }^{*}$ Corresponding author: Asghar Aghamohammadi, Research Center for Immunodeficiencies, Pediatrics Center of Excellence, Children's Medical Center, Tehran University of Medical \\ Sciences, Tehran, IR Iran. Tel: +98-2166428998, Fax:+98-2166923054, E-mail: aghamohammadi@sina.tums.ac.ir
}

Received 2015 July 26; Accepted 2015 November 30.

Keywords: Infection, Children, Pediatrics

\section{Dear Editor,}

X-linked hyper IgM syndrome (XHIGM or HIGM1) is a rare immunodeficiency disease caused by mutations in the gene that codes CD40 ligand (CD4OL), which is necessary for T cells to induce B cells to undergo immunoglobulin class-switching from IgM to IgG, IgA, and IgE (1). Thus, these patients have normal or elevated levels of IgM and markedly reduced levels of IgG, IgA, and IgE which leads to increased susceptibility to infections. Lymphoid tissue is very small because deficient CD4OL signaling does not activate B cells (2). Lymphocytic interstitial pneumonitis (LIP) is an uncommon disorder, especially in children, which is characterized by diffuse or multifocal reactive lymphoid infiltrate involving the alveolar interstitium. A number of diverse systemic illnesses are associated with LIP such as AIDS, Epstein-Barr virus infection, autoimmune disorders, and various primary immunodeficiency states including common variable immune deficiency (CVID) (2). The present case illustrated that LIP, rarely, can be a presentation of XHIGM syndrome. A 7-year old boy was admitted, on April 10, 2012, to the children medical center affiliated to Tehran University of Medical Sciences, Iran with clinical presentation of fever and nonproductive cough. Physical examination revealed fever ( $\mathrm{T}$ $=38.5^{\circ}$ auxiliary $)$, tachypnea $(\mathrm{RR}=35 / \mathrm{min})$, tachycardia $(\mathrm{PR}=120 / \mathrm{min})$, and respiratory distress. Lung auscultation revealed diffuse bilateral crackles. He also had hepatomegaly and digital clubbing. Examination of throat revealed normal size tonsils. Past medical history was not significant except one episode of pneumonia during the previous year. His parents are first-degree relative. A study of his family history showed that his sister and an aunt had died in infancy with unknown pulmonary disease. On admission, Chest X-ray (CXR) showed bilateral diffuse alveolar shadow (Figure 1). Clinically we suspected mycoplasma infection, thus azithromycin was administered. On the 3rd day of admission, respiratory distress was aggravated, but CXR showed no changes. High resolution computed tomography (HRCT) of lung without media contrast showed mixed interstitial and alveolar opacities with diffuse interlobular and intralobular septal thickening in both lungs more pronounced in central areas and some small peripheral and central cysts suggesting LIP (Figure 2). Due to existence of refractory pneumonia and bilateral alveolar opacities, a CT-guided biopsy was performed and diffuse infiltration of the alveolar septa by dense collections of lymphocytes and plasma cells with granuloma formation was reported which was in favor of LIP. Laboratory data of differential cell blood counts were as follows: white blood cell (WBC): $21.53 \times 10^{3} / \mu \mathrm{l}$; neutrophils: 19\%; lymphocytes: 65\%; Hb: $16 \mathrm{~g} / \mathrm{dl}$; and platelets: 761 $\times 10^{3} / \mu \mathrm{l}$. The most important data resulted from immunological studies reported in Table 1. Moreover, HIV PCR test was negative and bronchoalveolar lavage (BAL) sample was negative for tuberculosis and fungal infection.

Mutation analysis revealed a point mutation of exon 1 (c.107 T > C, p.M36R) of the CD40L gene which was in favor of HIGM syndrome. Eventually, the case was diagnosed as HIGM syndrome and administration of intravenous immunoglobulin (IVIG) with a dose of $400 \mathrm{mg} / \mathrm{kg}$ was started. For treatment of his lung disease, prednisolone $(2 \mathrm{mg} /$

Copyright (C) 2016, Growth \& Development Research Center. This is an open-access article distributed under the terms of the Creative Commons Attribution-NonCommercial 4.0 International License (http://creativecommons.org/licenses/by-nc/4.0/) which permits copy and redistribute the material just in noncommercial usages, provided the original work is properly cited. 
$\mathrm{kg}$ (day) was administered. His respiratory symptoms were improved gradually and he was discharged after 2 weeks with Pneumocystis jiroveci of oral cotrimoxazole for prevention of Pneumocystis jiroveci pneumonia and advices for avoiding Cryptosporidium parvum infection. The prednisolone was tapered after 8 weeks. During 6 months followup, his health condition was good with normal growth without presence of any infections. There was no evidence of dyspnea or respiratory distress, digital clubbing was improved, and the pulmonary function test (PFT) and CXR returned to normal (Figure 2). This case report describes a 7 years old boy with the rare primary immunodeficiency disease of XHIGM syndrome who presented with refractory pneumonia. Appropriate clinical suspicion and more investigations led us to find LIP according to findings of HRCT of lung and histologic features of lung biopsy. Diagnosis of HIGM was based on the presence of low serum IgG levels, normal levels of IgM and IgA, defective antibody response to protein antigens, presence of B-lymphocytes in flow cytometry and genetic analysis. Patients with XHIGM syndrome have defective CD40L expression on their activated helper CD4+ T cells. This leads to defective B cell responses and the failure of immunoglobulin classswitching. These features are typified in these patients by a profound reduction or absence of isotype class-switched memory B cells (CD19+, CD27+, IgM-, IgD-) with low or absent secretion of IgG and IgA, and normal or elevated serum IgM levels $(2,3)$. Due to the impairment of T-cell function and macrophage activation, XHIGM patients are particularly prone to opportunistic infections with Pneumocystis jiroveci, Cryptosporidium, Toxoplasma gondii. There are few published reports of LIP in HIGM syndrome in the literature. However, it was reported in other immunodeficiency diseases such as CVID (4). LIP is characterized by a diffuse infiltrate of mature and immature lymphocytes, plasma cells, and histiocytes in the alveolar septa and pulmonary interstitium. Nodular formation of lymphocytes is commonly seen, occasionally with germinal centers. The cellular infiltrate is polyclonal, including both $\mathrm{B}$ and $\mathrm{T}$ cells, usually with a predominance of CD8 lymphocytes (5). Marked lymphoid hypertrophy is a clinical feature of HIGM with adenosine induced cystidine deaminase (AICDA)
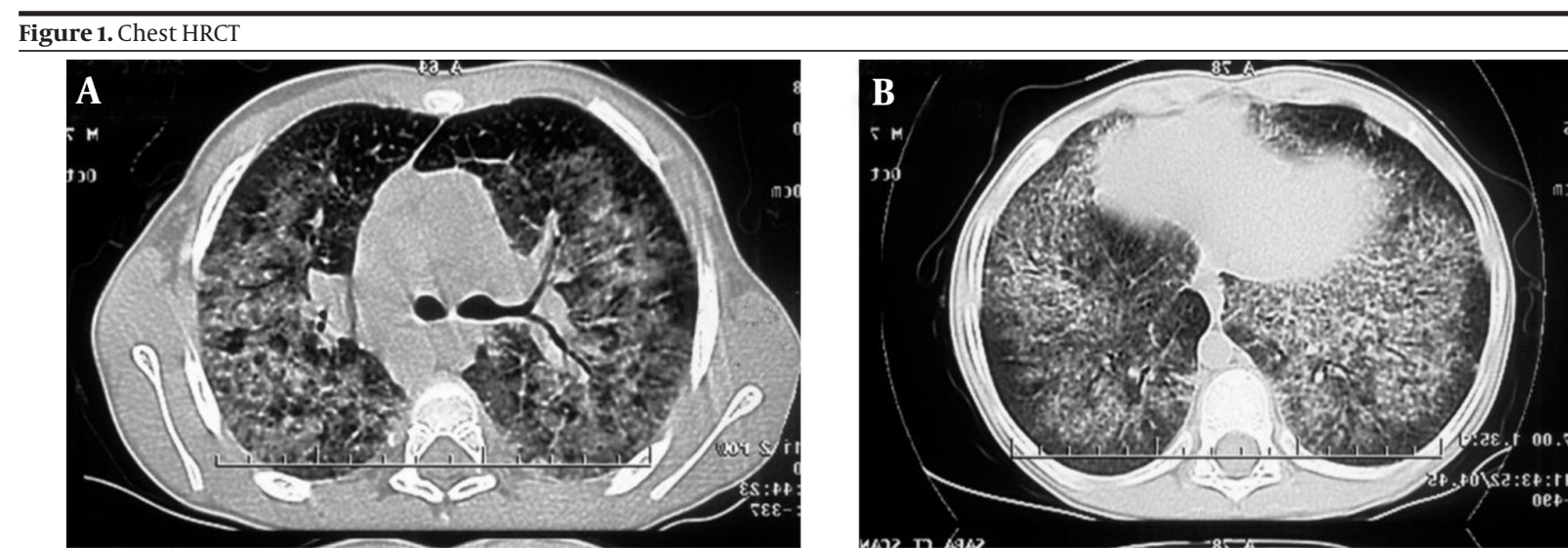

Mixed interstitial and alveolar opacities with diffuse interlobular and intra lobular septal thickening in both lungs more pronounced in central areas and some small peripheral and central cysts suggesting lymphocytic interstitial pneumonitis.

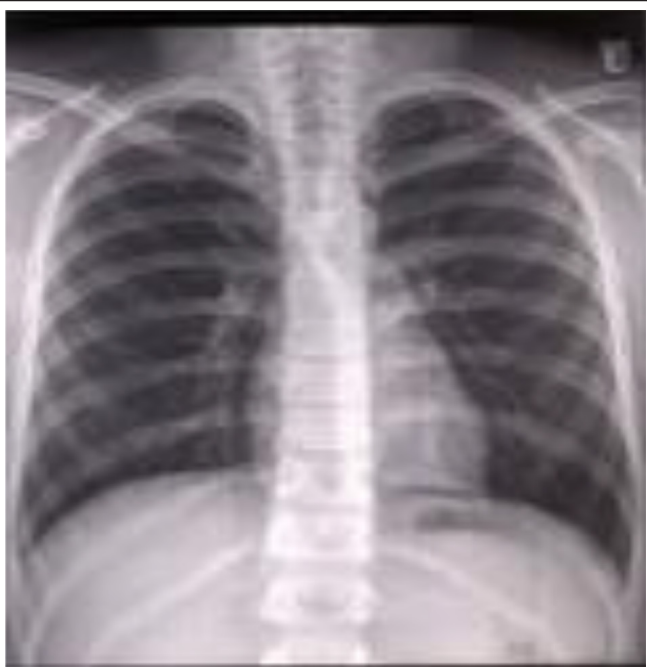

Figure 2. Normal Chest Radiography at the End of Treatment deficiency and mutation in uracil DNA glycosylase (UNG) gene (6). In our case, based on the presence of LIP and suspicious death of his sister and aunt, we suspected autosomal recessive form of HIGM with lymphoid hyperplasia such as AICDA and UNG gene mutation. Nevertheless, mutation investigation confirmed diagnosis of XHIGM syndrome. Lymphoid tissues in patients with XHIGM syndrome are devoid of germinal centers, because of the lack of activation of B cells by the T cells in them; however, lymphadenopathy may be present. In the European XHIGM Registry, generalized lymphadenopathy was reported in 7 of 56 patients (7). In this case, LIP can be an unusual presentation in HIGM, especially in XHIGM. In conclusion, in every immunocompromised patient with pulmonary involvement and refractory to conventional treatment, LIP should be considered in differential diagnosis for appropriate treatment. In these circumstances, a CT guided lung biopsy can reveal this entity. 
Reisi M et al.

Table 1. Immunological Data of the Patient With LIP at the Time of Diagnosis

\begin{tabular}{|c|c|c|}
\hline Parameters & Patient & Normal Range \\
\hline IgG (mg/dl) & 114 & $500-1300$ \\
\hline $\operatorname{IgA}(\mathrm{mg} / \mathrm{dl})$ & 169 & $41-297$ \\
\hline $\operatorname{IgE}(\mathrm{IU} / \mathrm{ml})$ & 9 & $\leq 90$ \\
\hline IgM (mg/dl) & 344 & $25-120$ \\
\hline Anti-tetanus (IU/ml) & $<0.01$ & $>0.1$ \\
\hline Anti-diphtheria (IU/ml) & $<0.01$ & $>0.1$ \\
\hline White blood cell count $\left(1^{3} / \mu l\right)$ & 21.53 & $4.5-13.5$ \\
\hline Lymphocytes count $\left(10^{3} / \mu \mathrm{l}\right)$ & 13.99 & $3-9.5$ \\
\hline CD3+ (\% of lymphocytes) & 61.8 & $30-78$ \\
\hline CD4+ (\% of lymphocytes) & 32.4 & $22-58$ \\
\hline CD8+ (\% of lymphocytes) & 28.3 & $10-37$ \\
\hline CD19+ (\% of lymphocytes) & 31.2 & $9-38$ \\
\hline CD56+ (\% of lymphocytes) & 5.3 & $3-15$ \\
\hline
\end{tabular}

\section{References}

1. Hirbod-Mobarakeh A, Aghamohammadi A, Rezaei N. Immunoglobulin class switch recombination deficiency type 1 or CD40 ligand deficiency: from bedside to bench and back again. Expert Rev Clin Immunol. 2014;10(1):91-105. doi 10.1586/1744666X.2014.864554. [PubMed: 24308834]

2. Etzioni A, Ochs HD. The hyper IgM syndrome-An evolving story. Pediatr Res. 2004;56(4):519-25. doi: 10.1203/01. PDR.000 0139318.65842.4A. [PubMed:15319456]

3. Etzioni A, Ochs HD. The hyper IgM syndrome--an evolving story. Pediatr Res. 2004;56(4):519-25. doi: 10.1203/01. PDR.000 0139318.65842.4A. [PubMed:15319456]

4. Davies CW, Juniper MC, Gray W, Gleeson FV, Chapel HM, Davies
RJ. Lymphoid interstitial pneumonitis associated with common variable hypogammaglobulinaemia treated with cyclosporin A. Thorax. 2000;55(1):88-90. [PubMed:10607809]

5. Swigris JJ. Lymphoid Interstitial Pneumonia*. CHEST Journal. 2002;122(6):2150. doi:10.1378/chest.122.6.2150.

6. Nilsen H, Stamp G, Andersen S, Hrivnak G, Krokan HE, Lindahl T, et al. Gene-targeted mice lacking the Ung uracil-DNA glycosylase develop B-cell lymphomas. Oncogene. 2003;22(35):5381-6. doi: 10.1038/sj.onc.1206860. [PubMed:12934097]

7. Levy J, Espanol-Boren T, Thomas C, Fischer A, Tovo P, Bordigoni P, et al. Clinical spectrum of X-linked hyper-IgM syndrome. J Pediatr.1997;131(1):47-54. doi:10.1016/s0022-3476(97)70123-9. 